\title{
Lepton Scattering, Hadrons and QCD
}


This page is intentionally left blank 

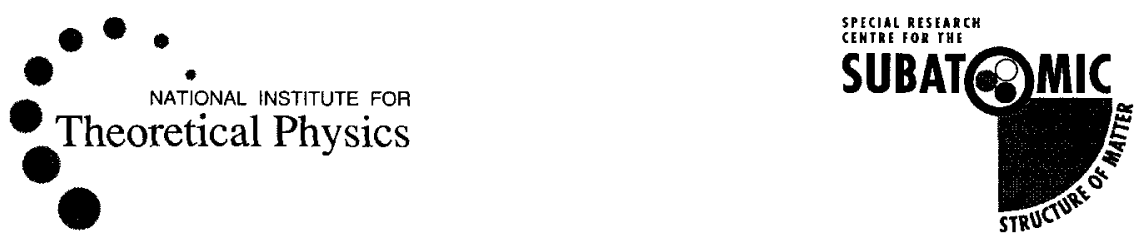

Proceedings of the Workshop on

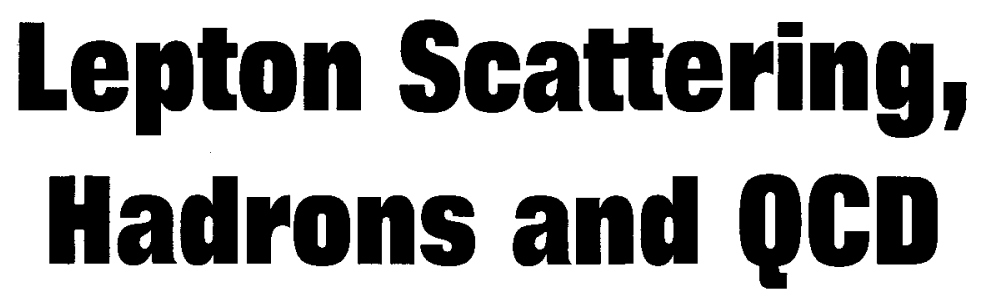

Adelaide, Australia $\quad 26$ March - 6 April 2001

Editors

W. Melnitchouk

Adelaide University, Australia Jefferson Laboratory, USA

A. W. Schreiber A. W. Thomas

Adelaide University, Australia

P. C. Tandy

Kent State University, USA 


\section{Published by}

World Scientific Publishing Co. Pte. Ltd.

P O Box 128, Farrer Road, Singapore 912805

USA office: Suite 1B, 1060 Main Street, River Edge, NJ 07661

UK office: 57 Shelton Street, Covent Garden, London WC2H 9HE

\section{British Library Cataloguing-in-Publication Data}

A catalogue record for this book is available from the British Library.

Front Cover: The cover shows the city of Adelaide at night, looking southeast across the River Torrens; this view was enjoyed by participants who attended the workshop banquet. The photo is used by courtesy of Tourism SA.

\section{LEPTON SCATTERING, HADRONS AND QCD}

Copyright $\odot 2001$ by World Scientific Publishing Co. Pte. Ltd.

All rights reserved. This book, or parts thereof, may not be reproduced in any form or by any means, electronic or mechanical, including photocopying, recording or any information storage and retrieval system now known or to be invented, without written permission from the Publisher.

For photocopying of material in this volume, please pay a copying fee through the Copyright Clearance Center, Inc., 222 Rosewood Drive, Danvers, MA 01923, USA. In this case permission to photocopy is not required from the publisher.

ISBN 981-02-4749-4

Printed in Singapore by Uto-Print 


\section{Foreword}

A group of about 65 physicists, from 9 countries, gathered in Adelaide in March 2001 to examine recent developments in the exploration of hadronic structure through lepton scattering and to advance the description of hadron physics directly from lattice QCD and non-perturbative QCD models.

This workshop was jointly organized and funded by the National Institute for Theoretical Physics (NITP) and Adelaide University's Special Research Centre for the Subatomic Structure of Matter (CSSM). Of the participants, 36 came from overseas and represented some 26 institutions in North America, Europe, and Japan. As with previous workshops hosted by the CSSM there were formal sessions of invited talks each morning and only one or two talks after coffee in the afternoons. Informal discussions and working sessions constituted the remainder of the time. As usual, this format was successful in stimulating considerable discussion and collaborative research.

The program covered the following topics: reviews of deep inelastic lepton scattering data; presentations of the most recent experimental results from DESY, HERA, SLAC, JLab, NIKHEF, and RIKEN; unpolarized and polarized structure functions, generalized parton distributions; nuclear effects; quarkhadron duality; electromagnetic form factors; structure functions and hadron properties from lattice QCD; models based on the Dyson-Schwinger equations. The prospects for small- $x$ physics at RHIC were also discussed. The speakers and topics were chosen in an effort to strengthen the links between these activities, and topics that attracted especial interest included the implications of chiral symmetry for lattice data, the first presentation of new $G_{E}^{p} / G_{M}^{p}$ data, leading protons/neutrons at DESY and quark-hadron duality.

On behalf of all the participants we would like to express our thanks to the group of people whose efforts enabled this workshop to run smoothly and ensured a stimulating atmosphere. Sharon Johnson and Sara Boffa of the CSSM office handled all of the logistics and the organizational matters with much generosity and skill; Ramona Adorjan provided very valuable professional assistance in computational matters.

All contributions in this volume have been independently refereed.

W. Melnitchouk

A. W. Schreiber P. C. Tandy

A. W. Thomas Adelaide, July 2001 
This page is intentionally left blank 


\section{Contents}

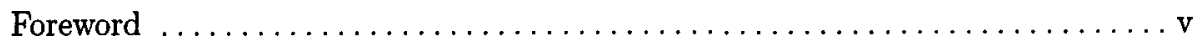

\section{PARTONIC STRUCTURE OF HADRONS}

Nucleon Form Factors and Structure Functions from Lattice QCD

M. Göckeler, R. Horsley, D. Pleiter, P. E. L. Rakow and G. Schierholz ....... 3

Chiral Extrapolation of Lattice Structure Function Calculations

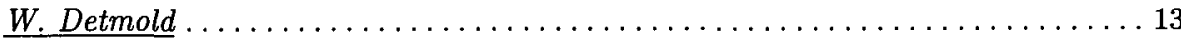

Charge Symmetry in Parton Distributions

J.T. Londergan

Distortions in the Negative Energy Dirac sea: Violation of the Gottfried Sum Rule and $\Delta \bar{u}$ in the Proton

K. Tsushima, A. W. Thomas and G. V. Dunne...................29

Parton Distributions for the Pion in a Chiral Quark Model

E. Ruiz Arriola ...................................... 37

Generalized Parton Distributions and Distribution of Partons in the

Transverse Plane

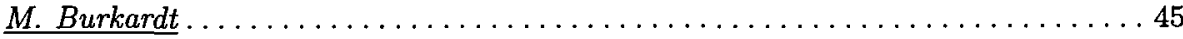

Exclusive Processes at HERMES

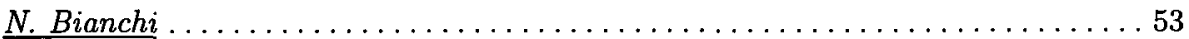

Soft Pion Production Associated with Deeply Virtual Compton Scattering

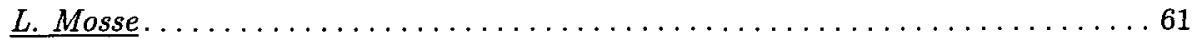

\section{SPIN STRUCTURE OF HADRONS}

An Experimental Review of the Nucleon Spin Structure Functions

G. G. Petratos.

Polarized Structure Functions in QCD

J. Kodaira 
viii

Single Spin Asymmetries and Quark Fragmentation

M. Anselmino, D. Boer, U. D'Alesio and F. Murgia

Determining $\Delta G$ with Polarized Photo- and Hadroproduction of Heavy Quarks

I. Bojak

Flavour Symmetry Breaking in the Polarized Nucleon Sea

F.-G. Cao and A. I. Signal. 102

\section{PERTURBATIVE - NONPERTURBATIVE QCD TRANSITION}

Lepton Scattering and Quark-Hadron Duality Studies at JLab

R. Ent.

Quark-Hadron Duality in Inclusive Electron-Hadron Scattering W. Melnitchouk. .

Signals of Local Duality from a Perturbative QCD Analysis of Inclusive ep Scattering

S. Liuti.

Gluons, Quarks and the Transition from Nonperturbative to Perturbative QCD

A. G. Williams

Estimating Low Energy Model Parameters from Deep Inelastic Scattering

L. P. Hoyt and A. I. Signal

\section{FORM FACTORS}

Measurement of $G_{E_{p}} / G_{M_{p}}$ to $Q^{2}=5.6 \mathrm{GeV}^{2}$ via Recoil

Polarization at Jefferson Lab

O. Gayou

Physical Hadron Properties from Lattice Data at Large Quark Masses

A. W. Thomas 165 
Meson Cloud Considerations in the Strange Magnetic Moment of the Nucleon from Lattice QCD

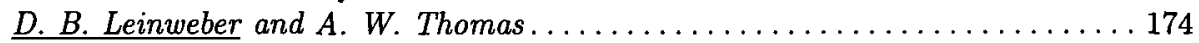

Electromagnetic Interactions in Light Front Dynamics

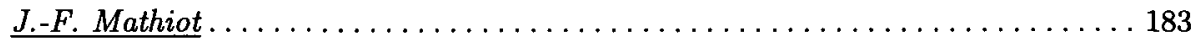

Soft QCD Modeling of Meson Electromagnetic Form Factors

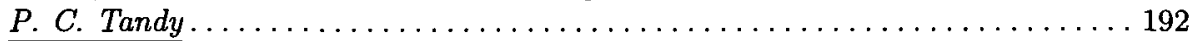

Nucleon Form Factors in the Covariant Diquark-Quark Model

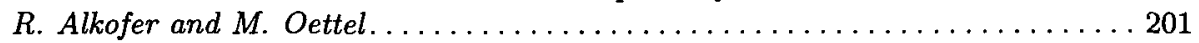

\section{HADRON EXCITATIONS, CONFINEMENT AND CHIRAL SYMMETRY BREAKING}

Experimental Studies of the Hadron Spectrum

J. Napolitano.

The Character of Goldstone Bosons

M. B. Hecht, C. D. Roberts and S. M. Schmidt

Confinement from Coulomb Gauge QCD and Exotic Phenomenology

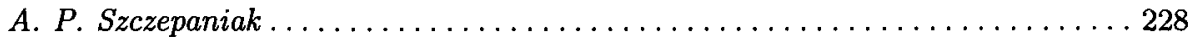

Regulator Free Dyson-Schwinger Equation Studies of Non-Perturbative

Field Theory

A. Kuzalersü, A. W. Schreiber, T. Sizer and A. G. Williams .......... 236

Domain-Like Structures in the QCD Vacuum, Confinement and Chiral

Symmetry Breaking

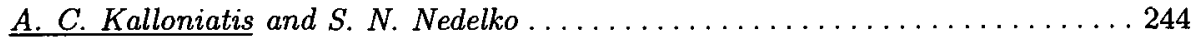

Glueball Properties in Anisotropic SU(3) Lattice QCD with an

Improved Action

N. Ishii, H. Suganuma and H. Matsufuru

Deconfining by Winding

R. Hofmann. 
Charmonium, Glueballs and Exotic Hybrids in a Relativistic Many-Body Approach

S. R. Cotanch

\section{SMALL- $x$ PHYSICS AND NUCLEAR MEDIUM EFFECTS}

Small $x$ Physics and the Initial Conditions in Heavy Ion Collisions

A. Krasnitz and $R$. Venugopalan ........................... 281

Leading Nucleon Production at HERA

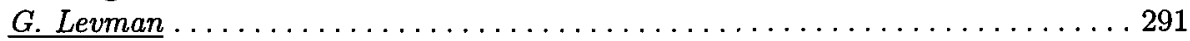

Nuclear Medium Effects at HERMES

P. di Nezza ..................................... 299

Non-Singlet Structure Function of the ${ }^{3} \mathrm{He}-{ }^{3} \mathrm{H}$ System and

Divergence of the Gottfried Integral

V. Guzey, A. W. Thomas, K. Tsushima, K. Saito and M. Strikman .... . 307

Quark-Diquark Structure of the Nucleon: Structure Functions,

Static Properties and Nuclear Matter Equation of State

W. Bentz, H. Mineo, A. W. Thomas and K. Yazaki ................... 314

Physics Motivation for a Polarized Electron-Ion Collider

J. M. Cameron and J. T. Londergan

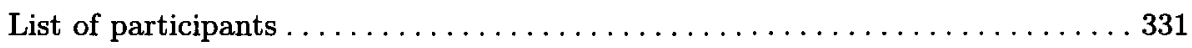

